\title{
The role of transcranial Doppler ultrasonography in differential diagnosis of vertigo in the Emergency Department
}

\author{
Antonio Siniscalchi, ${ }^{1}$ Giovanni Malferrari² \\ ${ }^{1}$ Stroke Unit, Department of Neurology, “Annunziata” Hospital, Cosenza; ${ }^{2}$ Stroke Unit, Department of \\ Neurology, Local Health Authority - IRCCS, Reggio Emilia, Italy
}

\begin{abstract}
Vertigo represents about $4 \%$ of access to the Emergency Department (ED). Several conditions, such as general medical illnesses, otovestibular diseases and neurological diseases (including posterior circulation stroke) cause acute vertigo. The incidence of cerebrovascular disease in patients with vertigo in ED varies from 3 to $5 \%$. Although neurosonology studies on acute vestibular syndrome are scarce, the use of transcranial Doppler (TCD) and transcranial color Doppler (TCCD) in the management of vertigo patients has several advantages: it can be performed at the patient's bedside and repeated and, furthermore, its use is low-cost. In an acute stroke, with an experienced doctor, it can help distinguish an ischemic stroke from a hemorrhagic stroke. In acute central vertigo induced by posterior circulation stroke, the TCD or TCCD can be a screening test before angiographic neuroradiological studies for stenosis of vertebral or basal arteries. As a matter of fact, the clinical outcome, particularly in the posterior circulation stroke, is mainly related to a rapid diagnosis and subsequent treatment that will be able to quickly restore the blood flow. In conclusion, TCD and TCCD are useful in the differential diagnosis of vertigo in the $\mathrm{ED}$, although we recognize the indisputable importance of clinical examination as a first step in vertigo management. In the evaluation of patients with acute central vertigo due to suspected posterior circulation stroke, the use of TCD or TCCD can rapidly reveal steno-occlusive disease of the posterior circulation, arterial dissections and give indirect signs of vertebra-basilar insufficiency.
\end{abstract}

Correspondence: Antonio Siniscalchi, Stroke Unit, Department of Neurology, "Annunziata" Hospital, Via F. Migliori 1, 87100 Cosenza, Italy.

Tel. +39.0984.681351 - Fax: +39.0984.21631.

E-mail: anto.siniscalchi@libero.it

Key words: Vertigo; Emergency Department; Posterior circulation stroke; Transcranial Doppler; Transcranial color Doppler.

Conflict of interest: the authors declare no potential conflict of interest.

Funding: none.

Received for publication: 11 June 2019.

Revision received: 16 July 2019.

Accepted for publication: 8 August 2019.

This work is licensed under a Creative Commons Attribution 4.0 License (by-nc 4.0).

(C) Copyright: the Author(s), 2019

Licensee PAGEPress, Italy

Emergency Care Journal 2019; 15:8341

doi:10.4081/ecj.2019.8341

\section{Introduction}

Vertigo is the illusory sense of movement, while unsteadiness is the feeling of being unstable while seated, standing, or walking. ${ }^{1}$ These symptoms represent the $4 \%$ of the access to the Emergency Department $(\mathrm{ED})^{2,3}$ and they can be related to both benign diseases of the inner ear ${ }^{4}$ or serious central nervous system diseases, including posterior circulation stroke. , $^{, 5}$

In this review, we provide an overview of clinical signs vertigo induced by posterior circulation stroke, the use of neuroradiological examinations and the use of transcranial Doppler (TCD) and transcranial coded color Doppler (TCCD) in the management of patients with vertigo.

\section{Methods of research}

PubMed, Embase, Cochrane library and reference lists were searched for articles published until May 30, 2019 using the keywords: vertigo, insufficiency vertebrobasilar, posterior circulation stroke, transcranial Doppler, transcranial color-coded Doppler.

\section{Vertigo in the Emergency Department}

Several conditions cause acute vertigo such as general medical diseases (including those not related to the cardiovascular system) (about 50\%), otovestibular diseases (about 33\%) and neurological diseases (including stroke) (about 11\%). ${ }^{6}$ In a prospective singleinstitution study, Cheung et al. ${ }^{7}$ reported that 23 of 413 patients (5.6\%) referred to the emergency department for dizziness have a diagnosis of central nervous system diseases. Similarly, Navi et $a l .{ }^{8}$ documented that 49 of 907 patients referred to ED for vertigo (5\%) have severe neurological disease; 37 of these were cerebrovascular events.

In a retrospective study conducted in Germany on 475 patients with vertigo referred to $\mathrm{ED}$, neurologists diagnosed a benign neurological disease in $73 \%$ of cases and a severe neurological disease (e.g. cerebrovascular diseases and inflammatory disease) in $27 \%$ of cases. ${ }^{9}$ The most common causes of central vertigo are vertebrobasilar insufficiency, stroke, transient ischemic attack (TIA), migraine, multiple sclerosis, posterior fossa tumors, neurodegenerative disorders, drugs and psychiatric conditions..$^{10}$ Central vertigo in stroke of posterior circulation may be accompanied by neurological symptoms such as ataxia, dysarthria, diplopia and visual weakness. ${ }^{11}$ Previous clinical studies suggested that some tests can be used to obtain a differential diagnosis between peripheral and central vertigo: i) gaze test; ii) alternative coverage test; iii) head impulse test; iv) targeted neurological examination, focusing on cranial nerves (including hearing), on cerebellar test and on longdistance signs; and v) gait test. ${ }^{3}$ The gait test is more important, in 
presence of central vertigo, even if for severely nauseated patients and too symptomatic for walking, is difficult verify the truncal ataxia. Patients requiring assistance for walk or sit, are more likely to have a central stroke (or other central nervous system disease) more than a vestibular neuritis. ${ }^{3}$ The tandem gait test evaluates the function of midline cerebellar, but in elderly people as well as in patients with peripheral vertigo it can give an uncorrected score. However, in vertigo patients, misdiagnosis can be also related to both inaccurate clinical evaluation, ${ }^{12}$ and a score of zero to the National Institute of Health Scale of stroke (NIHSS) $)^{13-15}$ as well as an excessive dependence of neuroradiological tests, such as computed tomography $(\mathrm{CT}) .{ }^{3}$ In a clinical study, a series of questions were asked to patients with vertigo to determine the reliability and consistency of eliminating the quality of symptoms and the timing and triggers of vertigo. ${ }^{16}$ When the main question was asked again 6 minutes later, half of the patients changed the type of primary vertigo, ${ }^{17}$ reducing the logic of a diagnostic process based on the type of vertigo. ${ }^{3}$ Patients with cardiovascular vertigo describe vertigo in almost $40 \%$ of cases. Patients with benign paroxysmal positional vertigo, commonly elderly patients, often describe non-vertiginous vertigo. ${ }^{18,19}$ Despite these data, most physicians still use a quality symptom approach with vertigo patients regardless of timing and triggers. ${ }^{3}$ A masked neurologist at the initial ED visit changed the diagnosis during the follow-up in $44 \%$ of patients. Benign vestibular diagnoses were found to be erroneous in $58 \%$ $(n=21$ of 36$)$, including $17 \%(n=6$ of 36$)$ with missed ischemic stroke or TIA. The most common reason for misdiagnosis $(70 \%)$ was an evolution of the clinical course over time. ${ }^{9}$ This is an integral part of emergency medicine; initially ambiguous symptoms or signs evolve during the time. There has never been (and probably will never be) a direct confrontation between emergency doctors and neurologists who diagnose patients with vertigo in the same phase of treatment, and sometimes the diagnosis of dizziness is complicated, also for physicians with specialized training.

\section{Vertigo as posterior circulation stroke}

The incidence of cerebrovascular disease in patients presenting with vertigo in ED ranges from 3 to $5 \%$. $^{4}$ This disorder is sometimes associated with a failure to diagnose stroke $^{5}$ that could increase the mortality. ${ }^{20}$ In particular, previous studies showed that approximately $10 \%$ of patients with cerebellar stroke may present, at least initially, symptoms that can mimic vestibular neuritis. ${ }^{4,21}$ Casani et $a l .{ }^{22}$ reported that about $3 \%$ of patients with vertigo had a missed cerebellar stroke. Both younger age and vertebral dissection are risk factors for missed cerebellar stroke. In particular, strokes of the posterior circulation is related to minor deficits and to a NIHSS of zero and therefore is undiagnosed. ${ }^{11,13,15}$ Unfortunately, the diagnosis of basilar artery occlusion is often delayed, because clinical symptoms may be misinterpreted ${ }^{23,24}$ and not well quantify neurological deficits after acute posterior circulation stroke. ${ }^{13-15,25-27}$ This may result in a missed or a delayed thrombolytic treatment. ${ }^{11,13,25}$ In an emergency room of a university hospital and a community, 103 out of 465 patients with ischemic stroke were misdiagnosed. Of these, $33 \%$ revealed a stroke in the posterior circulation and $16 \%$ a stroke in the anterior circulation. ${ }^{2}$ Concerning a difficult diagnosis of the posterior circulation stroke, in addition to the sensitivity of the lower NIHSS score, ${ }^{13}$ also the sensitivity of CT is about 7-16\% in the first 24 hours and the sensitivity of magnetic resonance imaging (MRI) is about $10-20 \%$ during the first 24-48 hours after the onset of the disorder. ${ }^{29-32}$

\section{Diagnosis of vertigo by neuroimaging}

Patients with a suspected diagnosis of transient ischemic attacks or vertebrobasilar strokes should undergo neuroimaging testing. Magnetic resonance angiography (MRA) may be used to identify occlusions and stenosis in neck and in large intracranial vessels..$^{33}$ Nakagawa et al.,${ }^{34}$ evaluating patients with vertebrobasilar insufficiency and patients with non-sick controls and patients with other peripheral labyrinth diseases, documented that patients with vertebrobasilar insufficiency have focal atherosclerosis that occlude the lumen of the basilar artery (mainly when near the exit of the anterior inferior cerebellar artery, AICA) and the origin of the vertebral artery. Moubayed and Saliba ${ }^{35}$ reported that $85.7 \%$ of patients with dizziness and at least 3 risk factors for stroke have morphological abnormalities in the vertebral arteries. Kim et al. ${ }^{36}$ performed MRA in 935 patients reported that the prevalence of stenosis gradually increased based on the severity of the disease and the results were statistically significant $(\mathrm{P}<0.0001$ for each comparison performed). The authors also consider the proximal region of the vertebral arteries as the place with a greater prevalence of occlusion of the posterior circulation compared with those of the distal vertebral/basilar artery. Concerning the posterior circulation, studies on healthy patients indicate that angio-tomography and arteriography have a similar sensitivity and specificity to evaluate the basilar artery. ${ }^{37}$ With regard to vertebro-basilar circulation, other studies on healthy subjects have reported the sensitivity of MRA and transcranial Doppler to $93.9 \%, 100 \%$, respectively, while the specificity rate was $94.8 \%$ and $95.2 \% .{ }^{38,39}$

\section{Diagnosis of vertigo using transcranial Doppler}

TCD or TCCD is an inexpensive, painless and non-invasive test that can measure the speed and direction of blood flow from large intracranial arteries..$^{40-43}$ The use of TCD or TCCD has several advantages: it can be performed at the bedside and repeated if necessary or applied for continuous monitoring; its use also is less expensive. In an acute setting, with an experienced clinician, it can help distinguish an ischemic stroke from a hemorrhagic stroke. Neurosonological investigations can also indicate underlying stroke mechanisms (a diagnosis of macroangiopathic, cardioembolic stroke, in artery dissection; or raise suspicion on indirect signs of vascular malformations) and also used in cerebral hemodynamic monitoring. TCD or TCCD can be a screening test before angiographic studies. TCD also allows real-time evaluation of average flow velocity, pulsatility and microembolization, information that is not available with angiography. The limitations of TCD, such as dependence on indirect parameters to identify blood vessels, poor spatial resolution, failure to display anatomical landmarks, inaccurate blood velocity metrics and incorrect classification of specific blood vessels in the presence of normal anatomical variants are exceeded with the use of TCCD. ${ }^{44}$ Previous clinical studies have reported the usefulness of TCCD in highlighting the stenosis of large cerebral vessels in the presence of an acute ischemic stroke. ${ }^{41,45-49}$ Unconstrained TCD has been reported to have a sensitivity of $55-81 \%$ and a specificity of $96 \%$ compared to digital subtraction angiography in patients with basilar or vertebral artery occlusion, while the use of TCCD has both a sensitivity and specificity of $100 \%$ related to a stenosis of vertebral or basic arteries. ${ }^{43}$ Most of the data on ischemia in the posterior circulation derive from the posterior circulatory register of New England Medical Center ${ }^{50}$ consisting of a consecutive series of 407 patients 
with signs and symptoms of ischemia with posterior circulation observed during a 10-year period. Embolism was the most common stroke mechanism ( $40 \%$ of patients included $24 \%$ of cardiac origin, $14 \%$ intra-arterial, $2 \%$ cardiac and arterial). The infarcts included the distal posterior territory of the circulation (rostral brainstem, upper cerebellum and occipital and temporal lobes); the proximal (medulla and inferior part of the cerebellum) and middle (bridge and inferior anterior cerebellum) territories were equally involved. Serious occlusive lesions ( $>50 \%$ stenosis) involved more than one large artery in 148 patients; 134 had an arterial site involved unilaterally or bilaterally. The most common occlusive sites were: intracranial vertebral artery (40 patients, 12 bilateral), basilar artery (46 patients) and 6 patients had proximal vertebral arterial dissection. Intra-arterial embolism was the most common mechanism of cerebral infarction in patients with occlusive disease of the vertebral arteries. Thirty-day mortality was 3.6\%. Embolic mechanism, position of the distal territory and occlusive disease of the basilar artery have reported the worst prognosis. The use of ultrasound (TCD or TCCD) in the acute phase in the acute pathology of the posterior circulation is considerable diagnostic help due to the inherent characteristics of the vertebral-basilar circle by transtemporal and transforaminal windows. In the acute phase the sonological characteristics of steno-occlusion of the vertebro-basilar are easy to diagnose, while the sonological characteristics of occlusion of distal vessels such as the inferior cerebellar artery or the AICA are not always easily detected, because are terminal branches (Figure 1). Recently, Microvascularization (MicroV) is an emerging Power Doppler technology that directly assesses the degree of stenosis not only through velocitometric study but also through morphological study. ${ }^{42,51}$ Regarding the diagnosis of cerebral hemorrhage, several TCCD studies have shown that the detection of a homogeneously hyperechoic area, clearly delimited by the surrounding brain tissue, may be representative of acute intracerebral hemorrhage..$^{22}$ The TCCD can identify complications of stroke such as a hemorrhagic transformation, ventricular bleeding and to monitor the midline shift, ${ }^{44}$ avoiding repeating serial neuroradiological investigations. In patients with space-occupying infarcts, since the midline shift displacement may predict a fatal outcome in patients with malignant cerebral infarcts ${ }^{44}$ and differentiate between an intracerebral hemorrhage and an ischemic stroke with a sensitivity of $95 \%$ and a specificity of $94 \% . .^{53,54}$ Therefore, if a CT scan is not readily available, as a first approach to the patient the TCCD can help also in identifying patients with primary cerebral hemorrhage or secondary bleeding complications. The TCCD, not only assesses the hemorrhagic complications of stroke, but can assess the collateral circulation for acute ischemic stroke and in acute middle cerebral artery territory stroke may aim to determine the perfusion of cerebral microvascularization, estimate infarct size and ischemic penumbra with implications for thrombectomy. ${ }^{55}$ Furthermore, in patients with vertebral-artery occlusion-basilar, the use of TCCD, when complete recanalization was not obtained after treatment with recombinant intravenous tissue (r-tPA; alteplase), could reduce the time for a thrombectomy, anticipating and avoiding a neuroradiological investigation.

\section{Limitations of the transcranial Doppler}

Insufficient acoustic window is the major factor that may hinder the use of TCCD in the adults. This factor is more common in the elderly, in women and in non-Caucasian races. It represents 10$20 \%$ of the inconclusive tests before the introduction of ultrasound contrast agents that amplify the echo. The use of contrast agents has reduced the abandonment rate to less than $7 \% .^{56}$ The other limitation is that TCCD is operator-dependent. This can be mitigated by adequate staff training to improve dexterity and allow diagnosis and safe interventions based on TCD or TCCD.

\section{Conclusions}

Although cerebrovascular ultrasonography can provide useful information in patients with vertebrobasilar insufficiency, we recognize the unquestionable importance of clinical examination as the first step in vertigo management. Although neurosonology studies on acute vestibular syndrome are scarce, ${ }^{56}$ the transcranial Doppler ultrasonography examination is a useful diagnostic test in patients with suspected vertigo, particularly in an elderly population with a significant burden of cerebrovascular risk factors. There appears to be sufficient evidence for the inclusion of $\mathrm{TCD} / \mathrm{TCCD}$ in a standard stroke imaging protocol, especially in resource-poor settings. Its ability to provide complementary information on cerebral architecture and stroke vasculature serves as a real alternative to CT/MRI for critically ill patients, although low sensitivity ${ }^{57,58}$ presented limits its value as a screening tool, in particular following the limitation in the evaluation of the disease of small vessels. This technology competes fairly well with more developed CT/MRI technologies. Therefore, as no patient with a suspected stroke would be denied a form of neuroimaging, the use of transcranial Doppler should also be added. The clinical outcome, particularly in posterior circulation stroke, is mainly related to a rapid diagnosis and subsequent treatment that will be able to quickly restore the blood flow in the posterior circulation.

In conclusion, TCD and TCCD are useful in the evaluation of patients with acute vertigo, as they can highlight steno-occlusive disease of the posterior circulation, arterial dissections and give indirect signs of vertebrobasilar insufficiency, particularly in patients suspected of having an acute ischemic stroke.

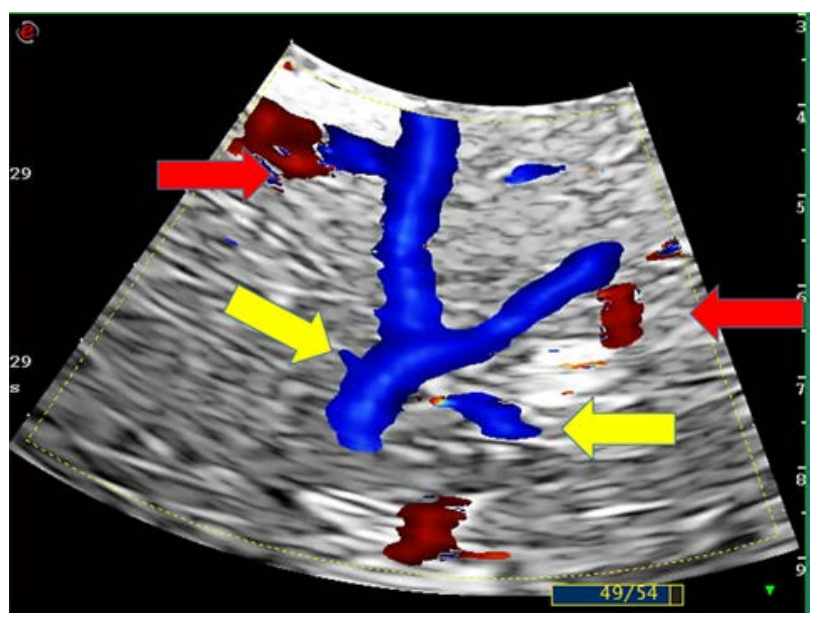

Figure 1. Transcranial color Doppler, transforaminal insonation plane: color-mode imaging of two vertebral arteries and proximal basilar artery. Red arrows correspond to the posterior inferior cerebellar arteries (PICA) arising laterally from the vertebral arteries; yellow arrows indicate anterior inferior cerebellar arteries (AICA) from the proximal basilar artery. 


\section{References}

1. Bisdor A, Von Brevern M, Lempert T, et al. Classification of vestibular symptoms: towards an international classification of vestibular disorders. J Vestib Res 2009;19:1-13.

2. Karatas M. Central vertigo and dizziness: epidemiology, differential diagnosis, and common causes. Neurologist 2008;14:355-64.

3. Edlow JA, Gurley KL, Newman-Toker DE. A new diagnostic approach to the adult patient with acute dizziness. J Emerg Med 2018;54:469-83.

4. Vanni S, Pecci R, Edlow JA, et al. Differential diagnosis of vertigo in the emergency department: a prospective validation study of the STANDING algorithm. Front Neurol 2017;8:590.

5. Savitz SI, Caplan LR, Edlow JA. Pitfalls in the diagnosis of cerebellar infarction. Acad Emerg Med 2007;14:63-8.

6. Newman-Toker DE, Hsieh YH, Camargo CA Jr, et al. Spectrum of dizziness visits to US emergency departments: cross-sectional analysis from a nationally representative sample. Mayo Clin Proc 2008;83:765-75.

7. Cheung CS, Mak PS, Manley KV, et al. Predictors of important neurological causes of dizziness among patients presenting to the emergency department. Emerg Med J 2010;27:517-21.

8. Navi BB, Kamel H, Shah MP, et al. Rate and predictors of serious neurologic causes of dizziness in the emergency department. Mayo Clin Proc 2012;87:1080-8.

9. Royl G, Ploner CJ, Leithner C. Dizziness in the emergency room: diagnoses and misdiagnoses. Eur Neurol 2011;66:25663.

10. Lee AT. Diagnosing the cause of vertigo: a practical approach. Hong Kong Med J 2012;18:327-32.

11. Nouh A, Remke J, Ruland S. Ischemic posterior circulation stroke: a review of anatomy, clinical presentations, diagnosis, and current management. Front Neurol 2014;5:30.

12. Kerber KA, Newman-Toker DE. Misdiagnosis of vertigo patients: common pitfalls in clinical practice. Neurol Clin 2015;33:565-75.

13. Siniscalchi A, Sztajzel R, Malferrari G, et al. The National Institutes of Health Scale of stroke: its role in patients with posterior circulation stroke. Hosp Top 2017;95:79-81.

14. Anticoli S, Bravi MC, Perillo G, et al. Effect of cardioembolic etiology on intravenous thrombolysis effectiveness for acute ischemic stroke. Curr Neurovasc Res 2016;13:193-8.

15. Siniscalchi A, Lochner P, Perrotta P, et al. Paralysis of isolated hand in the National Institutes of Health Travel Scale (NIHSS): Is it useful? West J Emerg Med 2018;19:524-6.

16. Newman-Toker DE. Charted records of dizzy patients suggest emergency physicians emphasize symptom quality in diagnostic assessment. Ann Emerg Med 2007;50:204-5.

17. Newman-Toker DE, Cannon LM, Stofferahn ME, et al. Imprecision in patient reports of dizziness symptom quality: a cross-sectional study conducted in an acute care setting. Mayo Clin Proc 2007;82:1329-40.

18. Newman-Toker DE, Dy FJ, Stanton VA, et al. How often is dizziness from primary cardiovascular disease true vertigo? A systematic review. J Gen Intern Med 2008;23:2087-94.

19. Lawson J, Johnson I, Bamiou DE, et al. Benign paroxysmal positional vertigo: clinical characteristics of dizzy patients referred to a Falls and Syncope Unit. QJM 2005;98:357-64.

20. Tarnutzer AA, Berkowitz AL, Robinson KA, et al. Does my dizzy patient have a stroke? A systematic review of bedside diagnosis in acute vestibular syndrome. CMAJ 2011;183:571-
92.

21. Newman-Toker D, Kattah J, Talkad A, et al. H.I.N.T.S. to diagnose stroke in the acute vestibular syndrome - three-step bedside oculomotor exam more sensitive than early MRI DWI. Stroke 2009;40:3504-10.

22. Casani AP, Dallan I, Cerchiai N, et al. Cerebellar infarctions mimicking acute peripheral vertigo: how to avoid misdiagnosis? Otolaryngol Head Neck Surg 2013;32:1518-21.

23. Masuda Y, Tei H, Shimizu S, et al. Factors associated with the misdiagnosis of cerebellar infarction. J Stroke Cerebrovasc Dis 2013;22:1125-30.

24. Siniscalchi A, Sztajzel R, Malferrari G, et al. Editorial: cocaine and acute basilar artery occlusion: what we know to date? Curr Vasc Pharmacol 2017;15:3-4.

25. Siniscalchi A, De Sarro G, Pacifici R, et al. Thrombolytic therapy in cocaine users with ischemic stroke: a review of current practice. Psychopharmacol Bull 2019;49:70-9.

26. Meyer BC, Hemmen TM, Jackson MC, et al. Modified National Institutes of Health Stroke Scale for use in stroke clinical trials: prospective reliability and validity. Stroke 2002;33:1261-6.

27. Pezzella FR, Pozzessere C, Siniscalchi A, et al. The cloud stroke unit: 24-hour acute stroke expertise-on-demand. Hosp Top 2013;91:81-6.

28. Arch AE, Weisman DC, Coca S, et al. Missed ischemic stroke diagnosis in the emergency department by emergency medicine and neurology services. Stroke 2016;47:668-73.

29. Chalela JA, Kidwell CS, Nentwich LM, et al. Magnetic resonance imaging and computed tomography in emergency assessment of patients with suspected acute stroke: a prospective comparison. Lancet 2007;369:293-8.

30. Choi JH, Kim HW, Choi KD, et al. Isolated vestibular syndrome in posterior circulation stroke: frequency and involved structures. Neurol Clin Pract 2014;4:410-8.

31. Kattah JC, Talkad AV, Wang DZ, et al. HINTS to diagnose stroke in the acute vestibular syndrome: three- step bedside oculomotor examination more sensitive than early MRI diffusion-weighted imaging. Stroke 2009;40:3504-10.

32. Saber Tehrani AS, Kattah JC, Mantokoudis G, et al. Small strokes causing severe vertigo: frequency of false-negative MRIs and non-lacunar mechanisms. Neurology 2014;83:16973.

33. Marx JJ, Mika-Gruettner A, Thoemke F, et al. Diffusion weighted magnetic resonance imaging in the diagnosis of reversible ischaemic deficits of the brain stem. J Neurol Neurosurg Psychiatry 2002;72:572-5.

34. Nakagawa T, Yamane H, Nakai Y, et al. Evaluation of the vertebrobasilar artery system by magnetic resonance angiography in the diagnosis of vertebrobasilar insufficiency. Acta Otolaryngol Suppl 1998;538:54-7.

35. Moubayed SP, Saliba I. Vertebrobasilar insufficiency presenting as isolated positional vertigo or dizziness: a double-blind retrospective cohort study. Laryngoscope 2009;119:2071-6.

36. Kim SH, Lee JS, Kwon OK, et al. Prevalence study of proximal vertebral artery stenosis using high-resolution contrast enhanced magnetic resonance angiography. Acta Radiol 2005;46:314-21

37. Graf J, Skutta B, Kuhn FP, Ferbert A. Computed tomographic angiography findings in 103 patients following vascular events in the posterior circulation: potential and clinical relevance. J Neurol 2000;247:760-6.

38. Khan S, Cloud GC, Kerry S, et al. Imaging of vertebral artery stenosis: a systematic review. J Neurol Neurosurg Psychiatry 
2007;78:1218-25.

39. Aaslid R, Markwalder TM, Nornes H. Noninvasive transcranial Doppler ultrasound recording of fow velocity in basal cerebral arteries. J Neurosurg 1982;57:769-72.

40. Rincon F, Sacco RL, Kranwinkel G, et al. Incidence and risk factors of intracranial atherosclerotic stroke: The Northern Manhattan Stroke Study. Cerebrovasc Dis 2009;28:65-71.

41. Malferrari G, Bertolino C, Casoni F, et al. The Eligible study: Ultrasound assessment in acute ischemic stroke within 3 hours. Cerebrovasc Dis 2007;24:469-76.

42. Malferrari G, Pulito G, Pizzini AM, et al. MicroV technology to improve transcranial color coded Doppler examinations. J Neuroimaging 2018;28:350-8.

43. Aaslid R, Markwalder TM, Nornes H. Noninvasive transcranial Doppler ultrasound recording of flow velocity in basal cerebral arteries. J Neurosurg 1982;57:769-74.

44. Bogdahn U, Becker G, Winkler J, et al. Transcranial colourcoded sonography in adults. Stroke 1990;21:1680-8.

45. Gerriets T, Postert T, Goertler M, et al. DIAS I: Duplex-sonographic assessment of the cerebrovascular status in acute stroke: A useful tool for future stroke trials. Stroke 2000;31:2342-5.

46. Allendoerfer J, Goertler M, von Reutern GM. Neurosonology in acute ischemic stroke study group: Prognostic relevance of ultra-early Doppler sonography in acute ischaemic stroke: a prospective multicenter study. Lancet Neurol 2006;5:835-40.

47. Kimura K, Yasaka M, Moriyasu H, et al. Ultrasonographic evaluation of vertebral artery to detect vertebrobasilar axis occlusion. Stroke 1994;25:1006-9.

48. Chi HY, Hsu CF, Chen AC, et al. Extracranial and intracranial ultrasonographic findings in posterior circulation infarction. $\mathrm{J}$ Ultrasound Med 2018;37:1605-10.

49. Min JH, Lee YS. Transcranial Doppler ultrasonographic evaluation of vertebral artery hypoplasia and aplasia. J Neurol Sci
2007;260:183-7.

50. Wityk RJ, Chang HM, Rosengart A, et al. Proximal extracranial vertebral artery disease in the New England Medical Center Posterior Circulation Registry. Arch Neurol 1998;55:470-8.

51. Malferrari G, Laterza D, Rossi S, et al. From velocimetry to morphology: a new classification for intracranial stenosis. "Overcoming Adam and Eve's sin". 24th Meeting of the European Society of Neurosonology and Cerebral Hemodynamics Joint Meeting with Neurosonology Research Group, 2019 Apr 5-7, Linz, Austria. pp 85-86.

52. Seidel G, Kaps M, Dorndorf W. Transcranial colour-coded duplex sonography of intracerebral haematomas in adults. Stroke 1993;24:1519-27.

53. Becker G, Hofmann E, Winkler J, et al. Differentiation between ischaemic and haemorrhagic stroke by transcranial colour-coded real-time sonography. J Neuroimaging 1993;3:42-7.

54. Mäurer M, Shambal S, Berg D, et al. Differentiation between intracerebral hemorrhage and ischemic stroke by transcranial color-coded duplex-sonography. Stroke 1998;29:2563-7.

55. Weismann M, Seidel G. Ultrasound perfusion imaging in human brain. Stroke 2000;31:2421-5.

56. Gerriets T, Goertler M, Stolz E, et al. Feasibility and validity of transcranial duplex sonography in patients with acute stroke. J Neurol Neurosurg Psychiatry 2002;73:17-20.

57. Tábuas-Pereira M, Sargento-Freitas J, Isidoro L, et al. Neurosonology accuracy for isolated acute vestibular syndromes. J Ultrasound Med 2017;36:2545-50.

58. Siniscalchi A, Gallelli L, Iannacchero R. Interictal cerebral posterior circulation in migraineurs with aura: a trancranial color-coded duplex sonography pilot study. J Headache Pain 2015;16:A159. 